\title{
On the representation of integers by indefinite binary Hermitian forms
}

\author{
Jouni Parkkonen Frédéric Paulin
}

October 29, 2018

\begin{abstract}
Given an integral indefinite binary Hermitian form $f$ over an imaginary quadratic number field, we give a precise asymptotic equivalent to the number of nonequivalent representations, satisfying some congruence properties, of the rational integers with absolute value at most $s$ by $f$, as $s$ tends to $+\infty$. 1
\end{abstract}

\section{Introduction}

Though less thoroughly developped than the real case of binary quadratic forms initiated by Gauss, the problem of the representation of integers by integral binary Hermitian forms, along with their reduction theory, initiated by Hermite, Bianchi and especially Humbert, has been much studied (see for instance [EGM1, Sect. 9] and references therein). The average, over the representatives of the binary Hermitian forms with a given discriminant, of the number of their nonequivalent representations of a given integer has been computed by Elstrodt, Grunewald, Mennicke [EGM2]. In this paper, we concentrate on a given form, and our result gives a precise asymptotic on the number of nonequivalent proper representations of rational integers with absolute value at most $s$ by a given integral indefinite binary Hermitian form.

A binary Hermitian form naturally gives rise to a quaternary quadratic form. The representations of integers by positive definite quaternary quadratic forms have been studied for a long time (including Lagrange's four square theorem, see also the work of Ramanujan as in $[\mathrm{Klo}$.) In the case of indefinite forms, the counting problem is complicated by the presence of an infinite group of automorphs of the form. General formulas are known (by Siegel's mass formula, see for instance [ERS]), but it does not seem to be easy (or even doable) to deduce our asymptotic formulae from them. Our proof is geometric, while the methods of EGM2 for the result quoted above rely on analytic techniques for the appropriate counting Dirichlet series. There are numerous results on counting integer points with bounded norm on quadrics, see for instance [dL, DRS, EM, BR] and the excellent survey [Bab]. In this paper, we consider a problem of a somewhat different nature, and we count appropriate orbits of integer points on which the form is constant.

Let $K$ be an imaginary quadratic number field, with discriminant $D_{K}$, ring of integers $\mathscr{O}_{K}$, and Dedekind zeta function $\zeta_{K}$. Fix an indefinite binary Hermitian form $f: \mathbb{C}^{2} \rightarrow \mathbb{R}$

\footnotetext{
${ }^{1}$ Keywords: Binary Hermitian form, representation of integers, group of automorphs, Bianchi group. AMS codes: 11E39, 11N45, 20H10, 30F40
} 
with

$$
f(u, v)=a|u|^{2}+2 \operatorname{Re}(b u \bar{v})+c|v|^{2}
$$

which is integral over $K$ (its coefficients satisfy $a, b \in \mathbb{Z}$ and $b \in \mathscr{O}_{K}$ ). The discriminant $\Delta(f)=|b|^{2}-a c$ of the form $f$ is positive. The group $\mathrm{SU}_{f}\left(\mathscr{O}_{K}\right)$ of automorphs of $f$ consists of those elements $g \in \mathrm{SL}_{2}\left(\mathscr{O}_{K}\right)$ for which $f \circ g=f$.

Let $\mathscr{P}_{K}$ be the set of relatively prime pairs of integers of $K$. For every $s>0$, the number of nonequivalent proper representations of rational integers with absolute value at most $s$ by $f$ is

$$
\psi_{f}(s)=\operatorname{Card} \mathrm{SU}_{f}\left(\mathscr{O}_{K}\right) \backslash\left\{(u, v) \in \mathscr{P}_{K}:|f(u, v)| \leq s\right\}
$$

The finiteness of $\psi_{f}(s)$ follows from general results on orbits of algebraic groups defined over number fields [AB, Lem. 5.3], see also [Shimu, Theo. 10.3].

In this paper, we prove the following theorem and its generalization (Corollary 66) for subsets of $\mathscr{P}_{K}$ satisfying additional congruence properties.

Theorem 1 As s tends to $+\infty$, we have the equivalent

$$
\psi_{f}(s) \sim \frac{\pi \operatorname{Covol}\left(\mathrm{SU}_{f}\left(\mathscr{O}_{K}\right)\right)}{2\left|D_{K}\right| \zeta_{K}(2) \Delta(f)} s^{2} .
$$

Note that the image of $\mathrm{SU}_{f}\left(\mathscr{O}_{K}\right)$ in $\mathrm{PSL}_{2}(\mathbb{C})$ is an arithmetic Fuchsian subgroup, and by definition, $\operatorname{Covol}\left(\mathrm{SU}_{f}\left(\mathscr{O}_{K}\right)\right)$ is the area of the quotient of the real hyperbolic plane $\mathscr{C}$ with constant curvature -1 preserved by $\mathrm{SU}_{f}\left(\mathscr{O}_{K}\right)$. The main input to prove Theorem 1 is the work $[\mathrm{PP}$ (building on [EM]), where we proved an equidistribution result for the boundary of big tubular neighbourhoods of a finite volume totally geodesic submanifold (here the image of $\mathscr{C}$ ) in the quotient of a real hyperbolic space by a lattice (here the Bianchi group $\operatorname{PSL}_{2}\left(\mathscr{O}_{K}\right)$ ).

The covolume of the group of automorphs could be computed using Prasad's very general formula in Pra]. Using the work of Maclachlan and Reid [Mac, MR1, MR2], building on results of Humbert and Vigneras, we give an expression for $\operatorname{Covol}\left(\operatorname{SU}_{f}\left(\mathscr{O}_{K}\right)\right)$ at the end of Section 2 (Remark 1).

As the final result of the note, we indicate how the results of [MR1] can be used to obtain an even more precise expression of the asymptotic formula in Theorem 1 when $K=\mathbb{Q}(i)$. A constant $\iota(f) \in\{1,2,3,6\}$ is defined as follows. If $\Delta(f) \equiv 0 \bmod 4$, let $\iota(f)=2$. If the coefficients $a$ and $c$ of the form $f$ as in Equation (11) are both even, let $\iota(f)=3$ if $\Delta(f) \equiv 1 \bmod 4$, and let $\iota(f)$ be the remainder modulo 8 of $\Delta(f)$ if $\Delta(f) \equiv 2$ $\bmod 4$. In all other cases, let $\iota(f)=1$.

Corollary 2 Let $f$ be an indefinite binary Hermitian form with Gaussian integral coefficients. Then as $s$ tends to $+\infty$,

$$
\psi_{f}(s) \sim \frac{\pi^{2}}{8 \iota(f) \zeta_{\mathbb{Q}(i)}(2)} \prod_{p \mid \Delta(f)}\left(1+\left(\frac{-1}{p}\right) p^{-1}\right) s^{2},
$$

where $p$ ranges over the odd positive rational primes and $\left(\frac{-1}{p}\right)$ is a Legendre symbol. 


\section{Representing integers by indefinite binary Hermitian forms}

Let $K, D_{K}, \mathscr{O}_{K}, \zeta_{K}, \mathscr{P}_{K}$ be as in the introduction, and let $\omega_{K}$ be the number of roots of unity in $\mathscr{O}_{K}$, that is, $\omega_{K}=4$ if $D_{K}=-4, \omega_{K}=6$ if $D_{K}=-3$ and $\omega_{K}=2$ if $D_{K} \neq-3,-4$. Let us first recall some facts about binary Hermitian forms.

The Lie group $\mathrm{SL}_{2}(\mathbb{C})$ acts linearly on the left on $\mathbb{C}^{2}$, and it acts on the right on the set of binary Hermitian forms $f$ by precomposition, that is by $f \mapsto f \circ g$ for every $g \in \mathrm{SL}_{2}(\mathbb{C})$, preserving the discriminant: $\Delta(f \circ g)=\Delta(f)$ for every $g \in \mathrm{SL}_{2}(\mathbb{C})$. The (nonuniform) lattice $\Gamma_{K}=\mathrm{SL}_{2}\left(\mathscr{O}_{K}\right)$ of $\mathrm{SL}_{2}(\mathbb{C})$ preserves the subset $\mathscr{P}_{K}$ of $\mathbb{C}^{2}$ as well as the set of integral indefinite binary Hermitian forms over $K$. The stabilizer in $\Gamma_{K}$ of such a form $f$ is the group of automorphs $\mathrm{SU}_{f}\left(\mathscr{O}_{K}\right)$ defined in the introduction.

For every indefinite binary Hermitian form $f$ as in Equation (11) with discriminant $\Delta=\Delta(f)$, let

and

$$
\begin{gathered}
\mathscr{C}_{\infty}(f)=\left\{[u: v] \in \mathbb{P}^{1}(\mathbb{C}): f(u, v)=0\right\} \\
\mathscr{C}(f)=\{(z, t) \in \mathbb{C} \times] 0,+\infty\left[: f(z, 1)+|a| t^{2}=0\right\} .
\end{gathered}
$$

Identifying, as usual, $\mathbb{P}^{1}(\mathbb{C})$ with $\mathbb{C} \cup\{\infty\}$, the set $\mathscr{C}_{\infty}(f)$ is the circle of center $-\frac{\bar{b}}{a}$ and radius $\frac{\sqrt{\Delta}}{|a|}$ if $a \neq 0$, and $\mathscr{C}_{\infty}(f)$ is the union of a real line with $\{\infty\}$ otherwise. The map $f \mapsto \mathscr{C}_{\infty}(f)$ induces a bijection between the set of indefinite binary Hermitian forms up to multiplication by a nonzero real factor and the set of circles and real lines in $\mathbb{C} \cup\{\infty\}$. The linear action of $\mathrm{SL}_{2}(\mathbb{C})$ on $\mathbb{C}^{2}$ induces a left action of $\mathrm{SL}_{2}(\mathbb{C})$ by homographies on the set of circles and real lines in $\mathbb{P}^{1}(\mathbb{C})$, and the map $f \mapsto \mathscr{C}_{\infty}(f)$ is anti-equivariant for the two actions of $\mathrm{SL}_{2}(\mathbb{C})$, in the sense that, for every $g \in \mathrm{SL}_{2}(\mathbb{C})$,

$$
\mathscr{C}_{\infty}(f \circ g)=g^{-1} \mathscr{C}_{\infty}(f) .
$$

Given a finite index subgroup $G$ of $\Gamma_{K}$, an integral binary Hermitian form $f$ is called $G$-reciprocal if there exists an element $g$ in $G$ such that $f \circ g=-f$. We define $R_{G}(f)=2$ if $f$ is $G$-reciprocal, and $R_{G}(f)=1$ otherwise. The values of $f(z, 1)$ are positive on one of the two components of $\mathbb{P}^{1}(\mathbb{C})-\mathscr{C}_{\infty}(f)$ and negative on the other. As the signs are switched by precomposition by an element $g$ as above, the reciprocity of the form $f$ is equivalent to saying that there exists an element of $G$ preserving $\mathscr{C}_{\infty}(f)$ and exchanging the two complementary components of $\mathscr{C}_{\infty}(f)$.

Theorem 1 will follow from the following more general result. For every finite index subgroup $G$ of $\Gamma_{K}$, let $G_{(1,0)}$ be the stabilizer of $(1,0) \in \mathbb{C}^{2}$ in $G$; let $\iota_{G}=1$ if - id $\in G$, and $\iota_{G}=2$ otherwise; for every $s>0$, let

$$
\psi_{f, G}(s)=\text { Card } \operatorname{SU}_{f}\left(\mathscr{O}_{K}\right) \cap G \backslash\{(u, v) \in G(0,1):|f(u, v)| \leq s\} .
$$

Taking $G=\Gamma_{K}$, which acts transitively on $\mathscr{P}_{K}$, we obtain the counting function $\psi_{f}=$ $\psi_{f, \Gamma_{K}}$ of the introduction. Note that the image of $\mathrm{SU}_{f}\left(\mathscr{O}_{K}\right) \cap G$ in $\mathrm{PSL}_{2}(\mathbb{C})$ is again an arithmetic Fuchsian subgroup. Let $\Gamma_{K, \infty}$ be the stabilizer of $[1: 0] \in \mathbb{P}^{1}(\mathbb{C})$ in $\Gamma_{K}$.

Theorem 3 Let $f$ be an integral indefinite binary Hermitian form over an imaginary quadratic number field $K$, and let $G$ be a finite index subgroup of $\mathrm{SL}_{2}\left(\mathscr{O}_{K}\right)$. Then as $s$ tends to $+\infty$, we have the equivalent

$$
\psi_{f, G}(s) \sim \frac{\pi \iota_{G}\left[\Gamma_{K, \infty}: G_{(1,0)}\right] \operatorname{Covol}\left(\operatorname{SU}_{f}\left(\mathscr{O}_{K}\right) \cap G\right)}{2 \omega_{K}\left|D_{K}\right| \zeta_{K}(2) \Delta(f)\left[\Gamma_{K}: G\right]} s^{2} .
$$


Proof. Let us first recall a geometric result from $[\mathrm{PP}$ that will be used to prove this theorem. A subset $A$ of a set endowed with an action of a group $G$ is precisely invariant under this group if for every $g \in G$, if $g A \cap A$ is nonempty, then $g A=A$.

Let $n \geq 2$ and let $\mathbb{H}_{\mathbb{R}}^{n}$ be the upper halfspace model of the real hyperbolic space of dimension $n$, with (constant) sectional curvature -1 . Let $F$ be a finite covolume discrete group of isometries of $\mathbb{H}_{\mathbb{R}}^{n}$. Let $1 \leq k \leq n-1$ and let $\mathscr{C}$ be a real hyperbolic subspace of dimension $k$ of $\mathbb{H}_{\mathbb{R}}^{n}$, whose stabilizer $F_{\mathscr{C}}$ in $F$ has finite covolume. Let $\mathscr{H}$ be a horoball in $\mathbb{H}_{\mathbb{R}}^{n}$, which is precisely invariant under $F$, with stabilizer $F_{\mathscr{H}}$.

For every $\alpha, \beta \in F$, denote by $\delta_{\alpha, \beta}$ the common perpendicular arc between $\alpha \mathscr{C}$ and the horosphere $\beta \partial \mathscr{H}$, and let $\ell\left(\delta_{\alpha, \beta}\right)$ be its length, counted positively if $\delta_{\alpha, \beta}$ exits $\beta \mathscr{H}$ at its endpoint on $\beta \partial \mathscr{H}$, and negatively otherwise. By convention, $\ell\left(\delta_{\alpha, \beta}\right)=-\infty$ if the boundary at infinity of $\alpha \mathscr{C}$ contains the point at infinity of $\beta \mathscr{H}$. Also define the multiplicity of $\delta_{\alpha, \beta}$ as $m(\alpha, \beta)=1 / \operatorname{Card}\left(\alpha F_{\mathscr{C}} \alpha^{-1} \cap \beta F_{\mathscr{H}} \beta^{-1}\right)$. For every $t \geq 0$, define $\mathscr{N}(t)=\mathscr{N}_{F, \mathscr{C}, \mathscr{H}}(t)$ as the number, counted with multiplicity, of the orbits under $F$ in the set of the common perpendicular $\operatorname{arcs} \delta_{\alpha, \beta}$ for $\alpha, \beta \in F$ with length at most $t$.

For every $m \in \mathbb{N}$, denoting by $\mathbb{S}_{m}$ the unit sphere of the Euclidean space $\mathbb{R}^{m+1}$, endowed with its induced Riemannian metric, we have the following result:

Theorem 4 ([PP, Coro. 4.9]) As $t \rightarrow+\infty$, we have

$$
\mathscr{N}(t) \sim \frac{\operatorname{Vol}\left(\mathbb{S}_{n-k-1}\right) \operatorname{Vol}\left(F_{\mathscr{H}} \backslash \mathscr{H}\right) \operatorname{Vol}\left(F_{\mathscr{C}} \backslash \mathscr{C}\right)}{\operatorname{Vol}\left(\mathbb{S}_{n-1}\right) \operatorname{Vol}\left(F \backslash \mathbb{H}_{\mathbb{R}}^{n}\right)} e^{(n-1) t}
$$

Let $f, K$ and $G$ be as in the statement of Theorem 3. We write $f$ as in Equation (11), and denote by $\Delta$ its discriminant. In order to apply Theorem 4 , we first define the various objects $n, k, F, \mathscr{H}$, and $\mathscr{C}$ that appear in its statement.

Let $n=3$ and $k=2$, so that $\operatorname{Vol}\left(\mathbb{S}_{n-1}\right)=4 \pi, \operatorname{Vol}\left(\mathbb{S}_{n-k-1}\right)=2$, the boundary at infinity of $\mathbb{H}_{\mathbb{R}}^{n}$ is $\partial \mathbb{H}_{\mathbb{R}}^{3}=\mathbb{C} \cup\{\infty\}$, and $\mathrm{PSL}_{2}(\mathbb{C})$ acts faithfully and isometrically on $\mathbb{H}_{\mathbb{R}}^{n}$ by the Poincaré extension of homographies.

For any subgroup $H$ of $\mathrm{SL}_{2}(\mathbb{C})$, we denote by $\bar{H}$ its image in $\mathrm{PSL}_{2}(\mathbb{C})$, except that the image of $\operatorname{SU}_{f}\left(\mathscr{O}_{K}\right)$ is denoted by $\operatorname{PSU}_{f}\left(\mathscr{O}_{K}\right)$. Let $F=\bar{G}$.

The Bianchi group $\overline{\Gamma_{K}}=\mathrm{PSL}_{2}\left(\mathscr{O}_{K}\right)$ acts discretely on $\mathbb{H}_{\mathbb{R}}^{3}$, with finite covolume. By a formula essentially due to Humbert (see for instance sections 8.8 and 9.6 of [EGM1]), we have

$$
\operatorname{Vol}\left(\overline{\Gamma_{K}} \backslash \mathbb{H}_{\mathbb{R}}^{3}\right)=\frac{1}{4 \pi^{2}}\left|D_{K}\right|^{3 / 2} \zeta_{K}(2) .
$$

Note that $\operatorname{Vol}\left(F \backslash \mathbb{H}_{\mathbb{R}}^{3}\right)=\left[\overline{\Gamma_{K}}: F\right] \operatorname{Vol}\left(\overline{\Gamma_{K}} \backslash \mathbb{H}_{\mathbb{R}}^{3}\right)$ and $\left[\overline{\Gamma_{K}}: \bar{G}\right]=\frac{1}{\iota_{G}}\left[\Gamma_{K}: G\right]$ by the definition of $\iota_{G}$.

Let $\mathscr{H}$ be the horoball centered at $\infty$ in $\mathbb{H}_{\mathbb{R}}^{3}$ that consists of all points in $\mathbb{H}_{\mathbb{R}}^{3}$ of Euclidean height at least 1 . This horoball is precisely invariant under $\overline{\Gamma_{K}}$ by Shimizu's lemma Shimi]. The stabilizer in the Bianchi group of the point at infinity $\infty$ is equal to $\left(\overline{\Gamma_{K}}\right) \mathscr{H}$. A fundamental domain $\Omega_{K}$ for the action of $\left(\overline{\Gamma_{K}}\right)_{\mathscr{H}}$ on $\mathbb{C}$ is

- the rectangle $[0,1] \times\left[0, \frac{\sqrt{\left|D_{K}\right|}}{2}\right]$ if $D_{K} \neq-3,-4$,

- the rectangle $[0,1] \times\left[0, \frac{1}{2}\right]$ if $D_{K}=-4$,

- and the parallelogram with vertices $0, \frac{1}{2}-\frac{i}{2 \sqrt{3}}, \frac{1}{2}+\frac{i}{2 \sqrt{3}}, \frac{i}{\sqrt{3}}$ if $D_{K}=-3$, 
see for instance [EGM1, page 318]. Since $\mathscr{F}_{K}=\Omega_{K} \times\left[1,+\infty\left[\subset \mathbb{H}_{\mathbb{R}}^{3}\right.\right.$ is a fundamental domain for the action of $\left(\overline{\Gamma_{K}}\right) \mathscr{H}$ on $\mathscr{H}$, an easy volume computation in hyperbolic geometry gives $\operatorname{Vol}\left(\mathscr{F}_{K}\right)=\frac{1}{2} \operatorname{Area}(\Omega)$. Hence by the values of the number $\omega_{K}$ of roots of unity,

$$
\operatorname{Vol}\left(\left(\overline{\Gamma_{K}}\right) \mathscr{H} \backslash \mathscr{H}\right)=\frac{\sqrt{\left|D_{K}\right|}}{2 \omega_{K}} .
$$

Note that $\operatorname{Vol}\left(\bar{G}_{\mathscr{H}} \backslash \mathscr{H}\right)=\left[\left(\overline{\Gamma_{K}}\right)_{\mathscr{H}}: \bar{G}_{\mathscr{H}}\right] \operatorname{Vol}\left(\left(\overline{\Gamma_{K}}\right) \mathscr{H} \backslash \mathscr{H}\right)$.

Let $\mathscr{C}=\mathscr{C}(f)$, which is indeed a real hyperbolic plane in $\mathbb{H}_{\mathbb{R}}^{3}$, whose set of points at infinity is $\mathscr{C}_{\infty}(f)$ (hence $\infty$ is a point at infinity of $\mathscr{C}(f)$ if and only if $a=0$ ). Note that $\mathscr{C}$ is invariant under the group $\mathrm{SU}_{f}\left(\mathscr{O}_{K}\right)$ by Equation (2) (which implies that $\mathscr{C}(f \circ g)=$ $g^{-1} \mathscr{C}(f)$ for every $\left.g \in \mathrm{SL}_{2}\left(\mathscr{O}_{K}\right)\right)$. The arithmetic group $\mathrm{SU}_{f}\left(\mathscr{O}_{K}\right)$ acts with finite covolume on $\mathscr{C}(f)$, its finite subgroup $\{ \pm \mathrm{id}\}$ acting trivially. By definition,

$$
\operatorname{Covol}\left(\operatorname{SU}_{f}\left(\mathscr{O}_{K}\right) \cap G\right)=\operatorname{Vol}\left(\operatorname{PSU}_{f}\left(\mathscr{O}_{K}\right) \cap F \backslash \mathscr{C}(f)\right)
$$

Note that $\operatorname{Covol}\left(\operatorname{SU}_{f}\left(\mathscr{O}_{K}\right) \cap G\right)$ depends only on the $G$-orbit of $f$, by Equation (2) and since $\mathrm{SU}_{f \circ g}\left(\mathscr{O}_{K}\right)=g^{-1} \mathrm{SU}_{f}\left(\mathscr{O}_{K}\right) g$ for every $g \in \mathrm{SL}_{2}\left(\mathscr{O}_{K}\right)$. By its definition, $R_{G}(f)$ is the index of the subgroup $\operatorname{PSU}_{f}\left(\mathscr{O}_{K}\right) \cap \bar{G}$ in $\bar{G}_{\mathscr{C}}$, hence

$$
\operatorname{Vol}\left(F_{\mathscr{C}} \backslash \mathscr{C}\right)=\frac{1}{R_{G}(f)} \operatorname{Covol}\left(\operatorname{SU}_{f}\left(\mathscr{O}_{K}\right) \cap G\right)
$$

Now that we have defined $n, k, F, \mathscr{H}$ and $\mathscr{C}$, let us pause in the proof, by recalling the following easy exercice in group theory.

Lemma 5 Let $C$ be a group and let $A, B, A^{\prime}, B^{\prime}$ be subgroups of $C$, such that $A \subset A^{\prime}$ and $B \subset B^{\prime}$, both with finite indices. Let $D$ be the set of elements $g \in C$ such that $g^{-1} A^{\prime} g \cap B^{\prime}=\{1\}$. Then the fibers of the canonical map from $A \backslash D / B$ to $A^{\prime} \backslash D / B^{\prime}$ all have cardinal $\left[A^{\prime}: A\right]\left[B^{\prime}: B\right]$.

Proof. Note that the subset $D$ of $C$, being invariant under left translation by $A^{\prime}$ and under right translation by $B^{\prime}$, is a disjoint union of double cosets $D=\coprod_{i \in I} A^{\prime} g_{i} B^{\prime}$. Write $A^{\prime}=\coprod_{j=1}^{m} A a_{j}$ and $B^{\prime}=\coprod_{k=1}^{n} b_{k} B$. Let us prove that $D=\coprod_{i \in I, 1 \leq j \leq m, 1 \leq k \leq n} A a_{j} g_{i} b_{k} B$, which yields the result. It is clear that $D$ is the union of the double cosets $A a_{j} g_{i} b_{k} B$. Let us prove that for every $a \in A$ and $b \in B$, if the equality $a a_{j} g_{i} b_{k} b=a_{j^{\prime}} g_{i^{\prime}} b_{k^{\prime}}$ holds, then $i=i^{\prime}, j=j^{\prime}, k=k^{\prime}$, which implies the disjointness of these double cosets. That equality implies first that $i=i^{\prime}$ by the definition of the double coset representatives $g_{i}$ 's, and thus that $g_{i}^{-1} a_{j^{\prime}}^{-1} a a_{j} g_{i}=b_{k^{\prime}} b^{-1} b_{k}^{-1}$. Since $a_{j^{\prime}}^{-1} a a_{j}$ and $b_{k^{\prime}} b^{-1} b_{k}^{-1}$ belong to $A^{\prime}$ and $B^{\prime}$ respectively, the assumptions defining $D$ imply that they are both equal to the identity element. Hence $a a_{j}=a_{j^{\prime}}$ and $b_{k} b=b_{k^{\prime}}$. By the definition of the left representatives $a_{j}$ 's and the right representatives $b_{k}$ 's, we hence have $j=j^{\prime}$ and $k=k^{\prime}$.

The last step of the proof of Theorem 3 consists in relating the two counting functions $\psi_{f, G}$ and $\mathscr{N}_{F, \mathscr{C}, \mathscr{H}}$, in order to apply Theorem 4 . For every $g \in \mathrm{SL}_{2}(\mathbb{C})$, let us compute the hyperbolic length of the common perpendicular arc $\delta_{g^{-1}}$,id between the real hyperbolic plane $g^{-1} \mathscr{C}$ and the horosphere $\partial \mathscr{H}$, assuming that they do not meet. The radius of the 
circle $\mathscr{C}_{\infty}(f \circ g)$, which is the boundary at infinity of $g^{-1} \mathscr{C}$ by Equation (2) , is $\frac{\sqrt{\Delta(f \circ g)}}{|a(f \circ g)|}$, where $a(f \circ g)$ is the coefficient of $|u|^{2}$ in $f \circ g(u, v)$. An immediate computation gives

$$
\ell\left(\delta_{g^{-1}, \mathrm{id}}\right)=\ln \frac{|a(f \circ g)|}{\sqrt{\Delta(f \circ g)}}=\ln \frac{|f \circ g(1,0)|}{\sqrt{\Delta(f)}} .
$$

With the conventions that we have taken, this formula is also valid if $g^{-1} \mathscr{C}$ and $\partial \mathscr{H}$ meet.

Let $U_{K}$ denote the stabilizer of $(1,0) \in \mathbb{C}^{2}$ in $\Gamma_{K}$, which consists of the unipotent upper triangular matrices in $\Gamma_{K}$. For every $s>0$, using Equation (3), we have

$$
\begin{aligned}
\psi_{f, G}(s) & =\operatorname{Card}\left\{[g] \in\left(\mathrm{SU}_{f}\left(\mathscr{O}_{K}\right) \cap G\right) \backslash G /\left(U_{K} \cap G\right):|f \circ g(1,0)| \leq s\right\} \\
& =\operatorname{Card}\left\{[g] \in\left(\operatorname{PSU}_{f}\left(\mathscr{O}_{K}\right) \cap \bar{G}\right) \backslash \bar{G} /\left(\overline{U_{K}} \cap \bar{G}\right): \ell\left(\delta_{g^{-1}, \text { id }}\right) \leq \ln \frac{s}{\sqrt{\Delta}}\right\} .
\end{aligned}
$$

Apply Lemma 5 to $C=\bar{G}=F, A=\operatorname{PSU}_{f}\left(\mathscr{O}_{K}\right) \cap \bar{G}, A^{\prime}=F_{\mathscr{C}}, B=\overline{U_{K}} \cap \bar{G}$ and $B^{\prime}=F_{\mathscr{H}}$. Note that there are only finitely many elements $[g] \in F_{\mathscr{C}} \backslash F / F_{\mathscr{H}}$ such that the multiplicity $m\left(g^{-1}\right.$, id $)=1 / \operatorname{Card}\left(g^{-1} F_{\mathscr{C}} g \cap F_{\mathscr{H}}\right)$ is different from 1 . Therefore, by the above and by Theorem 4 , we have

$$
\begin{aligned}
& \psi_{f, G}(s) \sim R_{G}(f)\left[\bar{G}_{\mathscr{H}}: \overline{U_{K}} \cap \bar{G}\right] \operatorname{Card}\left\{[g] \in F_{\mathscr{C}} \backslash F / F_{\mathscr{H}}: \ell\left(\delta_{g^{-1}, \mathrm{id}}\right) \leq \ln \frac{s}{\sqrt{\Delta}}\right\} \\
& \sim R_{G}(f)\left[\bar{G}_{\mathscr{H}}: \overline{U_{K}} \cap \bar{G}\right] \mathscr{N}_{F, \mathscr{C}, \mathscr{H}}\left(\ln \frac{s}{\sqrt{\Delta}}\right) \\
& \sim R_{G}(f)\left[\bar{G}_{\mathscr{H}}: \overline{U_{K}} \cap \bar{G}\right] \frac{2\left[\left(\overline{\Gamma_{K}}\right) \mathscr{H}: \bar{G}_{\mathscr{H}}\right] \frac{\sqrt{\left|D_{K}\right|}}{2 \omega_{K}} \frac{1}{R_{G}(f)} \operatorname{Covol}\left(\mathrm{SU}_{f}\left(\mathscr{O}_{K}\right) \cap G\right)}{4 \pi \frac{1}{\iota_{G}}\left[\Gamma_{K}: G\right] \frac{1}{4 \pi^{2}}\left|D_{K}\right|^{3 / 2} \zeta_{K}(2)} \frac{s^{2}}{\Delta},
\end{aligned}
$$

as $s$ tends to $+\infty$. Since $\left[\left(\overline{\Gamma_{K}}\right)_{\mathscr{H}}: \bar{G}_{\mathscr{H}}\right]\left[\bar{G}_{\mathscr{H}}: \overline{U_{K}} \cap \bar{G}\right]=\frac{1}{2}\left[\Gamma_{K, \infty}: G_{(1,0)}\right]$, Theorem 3 follows.

We now state the precise asymptotic result of the number of nonequivalent representations of rational integers, satisfying some congruence relations and having absolute value at most $s$, by a given integral indefinite binary Hermitian form. Given a nonzero ideal $\mathfrak{a}$ in $\mathscr{O}_{K}$, let $\iota_{\mathfrak{a}}=1$ if $2 \in \mathfrak{a}$, and $\iota_{\mathfrak{a}}=2$ otherwise; consider the congruence subgroups

$$
\Gamma_{K}(\mathfrak{a})=\left\{\left(\begin{array}{ll}
\alpha & \beta \\
\gamma & \delta
\end{array}\right) \in \Gamma_{K}: \alpha-1, \delta-1, \gamma, \beta \in \mathfrak{a}\right\}, \quad \Gamma_{K, 0}(\mathfrak{a})=\left\{\left(\begin{array}{ll}
\alpha & \beta \\
\gamma & \delta
\end{array}\right) \in \Gamma_{K}: \gamma \in \mathfrak{a}\right\} .
$$

Both $\Gamma_{K, 0}(\mathfrak{a})$ and $\Gamma_{K}(\mathfrak{a})$ coincide with $\Gamma_{K}$ when $\mathfrak{a}=\mathscr{O}_{K}$.

Corollary 6 Let $f$ be an integral indefinite binary Hermitian form over an imaginary quadratic number field $K$, and let $\mathfrak{a}$ be a nonzero ideal in $\mathscr{O}_{K}$. As s tends to $+\infty$, we have

$$
\begin{gathered}
\text { Card } \operatorname{SU}_{f}\left(\mathscr{O}_{K}\right) \cap \Gamma_{K}(\mathfrak{a}) \backslash\left\{(u, v) \in \mathscr{P}_{K}: u-1, v \in \mathfrak{a}, \quad|f(u, v)| \leq s\right\} \\
\sim \frac{\pi \iota_{\mathfrak{a}} \operatorname{Covol}\left(\mathrm{SU}_{f}\left(\mathscr{O}_{K}\right) \cap \Gamma_{K}(\mathfrak{a})\right)}{2 \mathrm{~N}(\mathfrak{a})^{2} \prod_{\mathfrak{p} \mid \mathfrak{a}}\left(1+\frac{1}{\mathrm{~N}(\mathfrak{p})}\right)\left|D_{K}\right| \zeta_{K}(2) \Delta(f)} s^{2},
\end{gathered}
$$

and

$$
\begin{gathered}
\text { Card } \operatorname{SU}_{f}\left(\mathscr{O}_{K}\right) \cap \Gamma_{K, 0}(\mathfrak{a}) \backslash\left\{(u, v) \in \mathscr{P}_{K}: v \in \mathfrak{a}, \quad|f(u, v)| \leq s\right\} \\
\sim \frac{\pi \operatorname{Covol}\left(\mathrm{SU}_{f}\left(\mathscr{O}_{K}\right) \cap \Gamma_{K, 0}(\mathfrak{a})\right)}{2 \mathrm{~N}(\mathfrak{a}) \prod_{\mathfrak{p} \mid \mathfrak{a}}\left(1-\frac{1}{\mathrm{~N}(\mathfrak{p})^{2}}\right)\left|D_{K}\right| \zeta_{K}(2) \Delta(f)} s^{2} .
\end{gathered}
$$


Proof. The orbits of $(1,0) \in \mathbb{C}^{2}$ under $\Gamma_{K}(\mathfrak{a})$ and $\Gamma_{K, 0}(\mathfrak{a})$ are precisely the sets $\{(u, v) \in$ $\left.\mathscr{P}_{K}: u-1, v \in \mathfrak{a}\right\}$ and $\left\{(u, v) \in \mathscr{P}_{K}: v \in \mathfrak{a}\right\}$ respectively.

The indices of $\Gamma_{K}(\mathfrak{a})$ and $\Gamma_{K, 0}(\mathfrak{a})$ in $\Gamma_{K}$, as computed for example in Theorems VII.16 and VII.17 of [New], are

$$
\left[\Gamma_{K}: \Gamma_{K}(\mathfrak{a})\right]=\mathrm{N}(\mathfrak{a})^{3} \prod_{\mathfrak{p} \mid \mathfrak{a}}\left(1+\frac{1}{\mathrm{~N}(\mathfrak{p})}\right) \quad \text { and } \quad\left[\Gamma_{K}: \Gamma_{K, 0}(\mathfrak{a})\right]=\mathrm{N}(\mathfrak{a}) \prod_{\mathfrak{p} \mid \mathfrak{a}}\left(1-\frac{1}{\mathrm{~N}(\mathfrak{p})^{2}}\right),
$$

where the products are taken over the prime ideals $\mathfrak{p}$ of $\mathscr{O}_{K}$ dividing $\mathfrak{a}$.

The index in $\Gamma_{K, \infty}$ of the stabilizer of $(1,0) \in \mathbb{C}^{2}$ in $\Gamma_{K}(\mathfrak{a})$ is $\omega_{K} \mathrm{~N}(\mathfrak{a})$. The index in $\Gamma_{K, \infty}$ of the stabilizer of $(1,0) \in \mathbb{C}^{2}$ in $\Gamma_{K, 0}(\mathfrak{a})$ is $\omega_{K}$.

Note that - id belongs to $\Gamma_{K, 0}(\mathfrak{a})$, but belongs to $\Gamma_{K}(\mathfrak{a})$ if and only if $2 \in \mathfrak{a}$, so that $\iota_{\Gamma_{K, 0}(\mathfrak{a})}=1$ and $\iota_{\Gamma_{K}(\mathfrak{a})}=\iota_{\mathfrak{a}}$.

The corollary now follows from Theorem 3, applied with $G=\Gamma_{K}(\mathfrak{a})$ and $G=\Gamma_{K, 0}(\mathfrak{a})$.

Theorem 1 follows from Corollary $\left[\right.$, by taking in both results $\mathfrak{a}=\mathscr{O}_{K}$. Note that from the techniques of [EGM1, Sect. 9], only the much weaker result $\psi_{f}(s)=O\left(s^{2} \log s\right)$ seems to be obtainable (see [EGM2, Coro. 2.12]).

In the following concluding remarks, for any positive integer $\Delta$, let

$$
f_{\Delta}(u, v)=|u|^{2}-\Delta|v|^{2},
$$

which is an integral indefinite binary Hermitian form with discriminant $\Delta$.

Remark 1. Here is a computation of $\operatorname{Covol}\left(\operatorname{SU}_{f}\left(\mathscr{O}_{K}\right)\right)$ for $f$ an integral indefinite binary Hermitian form over $K$, with discriminant $\Delta$, following [Mac, MR2] instead of [Pra].

Maclachlan has proved in $\mathrm{Mac}$ that $\mathrm{SU}_{f}\left(\mathscr{O}_{K}\right)$ and $\mathrm{SU}_{f_{\Delta}}\left(\mathscr{O}_{K}\right)$ are commensurable up to conjugation, in the following way. Since the limit set of $\operatorname{PSL}_{2}\left(\mathscr{O}_{K}\right)$ is $\partial \mathbb{H}_{\mathbb{R}}^{3}=\mathbb{C} \cup\{\infty\}$ and since $\mathrm{SU}_{f}\left(\mathscr{O}_{K}\right)=\mathrm{SU}-f\left(\mathscr{O}_{K}\right)$, we may assume, up to replacing $f$ by an element in its $\Gamma_{K^{-}}$ orbit or its opposite, that $a=a(f)>0$. Let $G_{a}$ be the congruence subgroup of $\operatorname{SU}_{f}\left(\mathscr{O}_{K}\right)$ which is the preimage of the upper triangular subgroup by the morphism $\operatorname{SU}_{f}\left(\mathscr{O}_{K}\right) \rightarrow$ $\mathrm{SL}_{2}\left(\mathscr{O}_{K} / a \mathscr{O}_{K}\right)$ of reduction modulo $a$ of the coefficients. Let $g=\left(\begin{array}{cc}\frac{1}{\sqrt{a}} & -\frac{\bar{b}}{\sqrt{a}} \\ 0 & \sqrt{a}\end{array}\right) \in \mathrm{SL}_{2}(\mathbb{C})$. By an easy computation, we have that $f \circ g=f_{\Delta}$, and that $g^{-1} G_{a} g$ is contained in $\operatorname{SL}_{2}\left(\mathscr{O}_{K}\right)$. Hence $g^{-1} G_{a} g$ is a finite index subgroup of $\operatorname{SU}_{f_{\Delta}}\left(\mathscr{O}_{K}\right)$. Therefore, we have

$$
\operatorname{Covol}\left(\mathrm{SU}_{f}\left(\mathscr{O}_{K}\right)\right)=\frac{\left[\mathrm{SU}_{f_{\Delta}}\left(\mathscr{O}_{K}\right): g^{-1} G_{a} g\right]}{\left[\mathrm{SU}_{f}\left(\mathscr{O}_{K}\right): G_{a}\right]} \operatorname{Covol}\left(\mathrm{SU}_{f_{\Delta}}\left(\mathscr{O}_{K}\right)\right)
$$

Maclachlan has also proved in $\mathrm{Mac}$ that $\mathrm{SU}_{f_{\Delta}}\left(\mathscr{O}_{K}\right)$ is commensurable with a lattice derived from a quaternion algebra, in the following way. Let $d_{K}=\frac{D_{k}}{4}$ if $D_{K} \equiv 0 \bmod 4$, and $d_{K}=D_{K}$ otherwise. Let $A$ be the quaternion algebra with Hilbert symbol $\left(\frac{d_{K}, \Delta}{\mathbb{Q}}\right)$ over $\mathbb{Q}$, which splits over $K$. Let $\Delta(A)$ be the reduced discriminant of $A$. Let $\mathscr{O}$ be the order $\mathbb{Z}[i, j, i j]$ in $A$ for the standard basis $1, i, j, i j$ of $A$, let $\mathscr{O}_{\max }$ be the maximal order containing $\mathscr{O}$, and let $\mathscr{O}^{1}, \mathscr{O}_{\max }^{1}$ be their groups of units. Let $\varphi: A \rightarrow A \otimes_{\mathbb{Q}} K=\mathscr{M}_{2}(K)$ be the natural embedding, given by

$$
\alpha+\beta i+\gamma j+\delta i j \mapsto\left(\begin{array}{cc}
\alpha+\beta \sqrt{d_{K}} & \Delta\left(\gamma+\delta \sqrt{d_{K}}\right) \\
\gamma-\delta \sqrt{d_{K}} & \alpha-\beta \sqrt{d_{K}}
\end{array}\right)
$$


An easy computation shows that $\varphi\left(\mathscr{O}^{1}\right)$ is a subgroup of $\mathrm{SU}_{f_{\Delta}}\left(\mathscr{O}_{K}\right)$. Then by MR2, Theo. 11.1.1], we have

$$
\operatorname{Covol}\left(\operatorname{SU}_{f_{\Delta}}\left(\mathscr{O}_{K}\right)\right)=\frac{\pi\left[\mathscr{O}_{\text {max }}^{1}: \mathscr{O}^{1}\right]}{3\left[\mathrm{PSU}_{f_{\Delta}}\left(\mathscr{O}_{K}\right): \overline{\varphi\left(\mathscr{O}^{1}\right)}\right]} \prod_{p \mid \Delta(A)}(p-1),
$$

where $p$ ranges over the positive rational primes. This gives a formula for $\operatorname{Covol}\left(\operatorname{SU}_{f}\left(\mathscr{O}_{K}\right)\right)$.

Remark 2. Assume that $K=\mathbb{Q}(i)$ in this remark. Let us give in this case, following [MR1, a more precise formula for $\operatorname{Covol}\left(\mathrm{SU}_{f}\left(\mathscr{O}_{K}\right)\right)$ where $f$ is an integral indefinite binary Hermitian form over $K$, with discriminant $\Delta$. Combined with Theorem 1 Corollary 2 will follow.

Since $\mathrm{SU}_{f}\left(\mathscr{O}_{K}\right)=\mathrm{SU}_{k f}\left(\mathscr{O}_{K}\right)$ for every $k \in \mathbb{N}-\{0\}$, we may assume, as required in MR1, that $f$ is primitive, that is, with $f$ as in Equation (1), the coefficients $a, c$, and the real and imaginary parts of $b$ have no common divisor in the rational integers. Note that the subgroup $\operatorname{PSU}_{f}\left(\mathscr{O}_{K}\right)$ of $\overline{\Gamma_{K}}$ is denoted by $\operatorname{Stab}\left(\mathscr{C}(f), \overline{\Gamma_{K}}\right)$ in [MR1, p. 161], and it is a maximal Fuchsian subgroup of $\overline{\Gamma_{K}}$ (loc. cit.).

The hyperbolic plane $\mathscr{C}\left(f_{\Delta}\right)$ associated to the form $f_{\Delta}$ is the halfsphere of Euclidean radius $\sqrt{\Delta}$ centered at 0 . A formula due to Humbert (see for instance [MR1, p. 169]) gives

$$
\operatorname{Covol}\left(\mathrm{SU}_{f_{\Delta}}\left(\mathscr{O}_{K}\right)\right)=\eta \pi \Delta \prod_{\substack{p \mid \Delta \\ p \text { odd }}}\left(1+\left(\frac{-1}{p}\right) p^{-1}\right)
$$

where $p$ ranges over the positive rational primes, $\left(\frac{-1}{p}\right)$ is a Legendre symbol, $\eta=1 / 2$ if $\Delta \equiv 0 \bmod 4$ and $\eta=1$ otherwise.

The maximal Fuchsian subgroups of $\overline{\Gamma_{K}}$ are classified in [MR1], yielding the following cases.

If $\Delta \equiv 0,3 \bmod 4$, then $\operatorname{PSU}_{f}\left(\mathscr{O}_{K}\right)$ is a conjugate in $\overline{\Gamma_{K}}$ of $\operatorname{PSU}_{f_{\Delta}}\left(\mathscr{O}_{K}\right)$, and its covolume is given by Equation (4) (see [MR1, p.170]).

If $\Delta \equiv 1 \bmod 4$, there are two cases: If the coefficients $a$ and $c$ are even, then

$$
\operatorname{Covol}\left(\operatorname{SU}_{f}\left(\mathscr{O}_{K}\right)\right)=\frac{1}{3} \operatorname{Covol}\left(\operatorname{SU}_{f_{\Delta}}\left(\mathscr{O}_{K}\right)\right)
$$

otherwise, $\operatorname{PSU}_{f}\left(\mathscr{O}_{K}\right)$ is a conjugate in $\overline{\Gamma_{K}}$ of $\operatorname{PSU}_{f_{\Delta}}\left(\mathscr{O}_{K}\right)$ (see [MR1, p. 172]).

If $\Delta \equiv 2 \bmod 4$, there are two cases: If the coefficients $a$ and $c$ are even, then

$$
\operatorname{Covol}\left(\operatorname{SU}_{f}\left(\mathscr{O}_{K}\right)\right)=\frac{1}{\eta^{\prime}} \operatorname{Covol~SU} f_{\Delta}\left(\mathscr{O}_{K}\right)
$$

where $\eta^{\prime} \in\{2,6\}$ satisfies $\eta^{\prime}=\Delta \bmod 8$; otherwise, $\operatorname{PSU}_{f}\left(\mathscr{O}_{K}\right)$ is a conjugate in $\overline{\Gamma_{K}}$ of $\operatorname{PSU}_{f_{\Delta}}\left(\mathscr{O}_{K}\right)$ (see [MR1, p. 173]).

This proves Corollary 2 of the introduction.

\section{References}

[AB] Harish-Chandra A. Borel. Arithmetic subgroups of algebraic groups. Ann. of Math. 75 (1962) 485-535. 
[Bab] M. Babillot. Points entiers et groupes discrets : de l'analyse aux systèmes dynamiques. in Rigidité, groupe fondamental et dynamique, Panor. Synthèses 13, 1-119, Soc. Math. France 2002.

[BR] M. Borovoi and Z. Rudnick. Hardy-Littlewood varieties and semisimple groups. Invent. Math. 119 (1995) 37-66.

[dL] D. de Lury. On the representation of numbers by the indefinite form $a x^{2}+b y^{2}+c z^{2}+d t^{2}$. Univ. Toronto Stud. Math. Ser. 5 (1938) 3-17.

[DRS] W. Duke, Z. Rudnick, and P. Sarnak. Density of integer points on affine homogeneous varieties. Duke Math. J. 71 (1993) 143-179.

[EGM1] J. Elstrodt, F. Grunewald, and J. Mennicke. Groups acting on hyperbolic space. Springer Verlag, 1998.

[EGM2] J. Elstrodt, F. Grunewald, and J. Mennicke. Zeta-functions of binary Hermitian forms and special values of Eisenstein series on three-dimensional hyperbolic space. Math. Ann. 277 (1987) 655-708.

[EM] A. Eskin and C. McMullen. Mixing, counting, and equidistribution in Lie groups. Duke Math. J. 71 (1993) 181-209.

[ERS] A. Eskin, Z. Rudnick, and P. Sarnak. A proof of Siegel's weight formula. Internat. Math. Res. Notices 5 (1991) 65-69.

[Klo] H. D. Kloosterman. On the representation of numbers in the form $a x^{2}+b y^{2}+c z^{2}+d t^{2}$. Acta Math. 49 (1927) 407-464.

[Mac] C. Maclachlan. Fuchsian subgroups of the groups $\mathrm{PSL}_{2}\left(O_{d}\right)$. In Low-dimensional topology and Kleinian groups (Coventry/Durham 1984), London Math. Soc. Lect. Note Ser. 112 158-181, Cambridge Univ. Press, 1986.

[MR1] C. Maclachlan and A. Reid. Parametrizing Fuchsian subgroups of the Bianchi groups. Canad. J. Math. 43 (1991) 158-181.

[MR2] C. Maclachlan and A. Reid. The arithmetic of hyperbolic 3-manifolds. Grad. Texts in Math. 219, Springer Verlag, 2003.

[New] M. Newman. Integral matrices. Academic Press, 1972.

[PP] J. Parkkonen and F. Paulin. Équidistribution, comptage et approximation par irrationnels quadratiques. Preprint [arXiv: 1004.0454].

[Pra] G. Prasad. Volumes of S-arithmetic quotients of semi-simple groups. Publ. Math. Inst. Hautes Études Sci. 69 (1989) 91-117.

[Shimi] H. Shimizu. On discontinuous groups operating on the product of half spaces. Ann. of Math. 77 (1963) 33-71.

[Shimu] G. Shimura. Arithmetic and analytic theories of quadratic forms and Clifford groups. Math. Surv. Monogr. 109, Amer. Math. Soc. 2004.

Department of Mathematics and Statistics, P.O. Box 35

40014 University of Jyväskylä, FINLAND.

e-mail: parkkone@maths.jyu.fi

DMA, UMR 8553 CNRS

Ecole Normale Supérieure, 45 rue d'Ulm

75230 PARIS Cedex 05, FRANCE

e-mail: Frederic.Paulin@ens.fr
Département de mathématique, Bât. 425

Université Paris-Sud 11

91405 ORSAY Cedex, FRANCE

e-mail: frederic.paulin@math.u-psud.fr 